\title{
Human Cytomegalovirus Infection as a Risk Factor for Type 2 Diabetes Mellitus Development in a Sample of Iraqi Patients
}

\author{
Huda Mohammed Hasan ${ }^{1}$, Dunya Fareed Salloom ${ }^{1}$ \\ ${ }^{1}$ Scholar Researchers, Biology Department College of Science, University of Baghdad, Iraq
}

\begin{abstract}
Type 2 diabetes mellitus which abbreviate as T2DM is a complex endocrine and metabolic disorder arising from genetic and environmental factors interaction which in turn induce various degrees of insulin functional alteration on peripheral tissues. Globally, T2DM has develop into a public health problem. Therefore, The study included (75) patients( 37 female and 38 males) suffering from T2DM who visit al-kadhimiya teaching hospital with age range 20-80 years and (70) as healthy controls with age range 20-70 years. All studied groups were evaluated CMV IgG by ELISA,B. urea, S. Creatinine, cholesterol and triglyceride the results showed that B.urea, S.creatinine and serum cholesterol showed a non-significant differences between studied group, While there was significant differences in concentration of triglyceride between both studied group. The seropositivity of CMV IgG was $62(82.87 \%)$ in T2DM patients with significant differences in compare to control.
\end{abstract}

Keyword: Type 2 Diabetes mellites, viral infection and Cytomegalovirus infection.

\section{Introduction}

The term T2DM derives from lack of the body ability to effectively use of insulin ${ }^{(1)}$. The risk factors for T2DM are genetic, obesity, family history for disease, sedentary lifestyle,ethnicity and other ${ }^{(2)}$. On the other hand, Environmental influences may also contribute in T2DM development and progression ${ }^{(1,2)}$. Moreover, T2DM consider as a low grade chronic inflammatory disorder with differences in function of immune cells ${ }^{(3-5)}$. The duration of diabetes is one of the strongest determinants of complication risk so, there was a positive correlation between FBS and duration of diabetes ${ }^{(6,7)}$. There is some proofs refer that chronic viral infections such as hepatitis B and HIV can increase susceptibility to chronic inflammation and immuno-metabolic responses and this result in T2DM development. The association between viral infection and diabetes may reflect an increased risk of pathogentic mechanism for some chronic viruses such

\footnotetext{
Corresponding Author:

Huda Mohammed Hasan

Scholar Researchers, Biology Department College of

Science, University of Baghdad, Iraq

e-mail: dunyascience@sc.uobaghdad.edu.iq
}

as HBV and HIV, resulting in insulin resistance linked to chronic inflammation ${ }^{\left({ }^{8}\right)}$. Some research also indicates that T2DM patients are more susceptible to incidence of viral infection since diabetes affects healing. Moreover, hyperglycemia frequently impairs coagulation, fibrin action, body fat and endothelium function (9). Cytomegalovirus like EBV since both of them belong to Herpes family can trigger autoimmunity and chronic inflammation ${ }^{(10)}$. The outstanding feature of latency for CMV caused unusual expansion of the CMV-specific resting effector population of memoryCD8 $+\mathrm{T}$ cell and thus leads to developed chronic inflammation and disturbed the host immune mechanisms ${ }^{(11-13)}$.

\section{Materials and Method}

Samples Investigated: A total sample of (145) Iraqi volunteers (75 T2DM patients: 38 males and 37 females compared to 70 healthy control individuals: 35 females and 35 males) were enrolled in a case-control investigation during the period from November 2019 to February 2020 after obtaining the approval of Ethical Committee at the University of Baghdad, College of Sciences, Biology Department and the Iraqi Ministry of Health. The written informed consent was possessed by all volunteers. The study was accomplished in accordance with the Ethics Code of the World Medical 
Association (Declaration of Helsinki) (World Health Organization 2013).

Detection of CMV IgG Antibody by ELISA: The detection of CMV IgG class of Ab in serum of human is relied on the technique of ELISA according to manufacture company human Germany.

Statistical Analysis: The data of CMV IgG serum level, age, gender, blood urea, S.creatinine, cholesterol and s. TG was analyzed for linearity, homogeneity and normal distribution using IBM SPSS statistical package version 26.0 (IBM Corp. Released 2019). The mean, standard error and the probability were calculated to determine the statistically significant differences.

\section{Results and Discussion}

One hundred forty five volunteers were incorporated in this study included seventy five T2DM patients and seventy healthy individuals considered as control group. Diabetic patients group included 38 males and 37 females' patients who were already diagnosed by laboratory test (FBS). In contrast, control group included 35 males and 35 females' participant. The present results showed a non-significant difference between T2DM group age mean and controls (Table 1). Also, the current study included T2DM patients with age range $20-80$ years, the highest age group was at the $41-50$ years age group $(34.55 \%)$, followed by $51-60$ years age group $(29.1 \%)$. While the lowest age group was at $20-30$ years $(3.6 \%)$.

Table 1: Demographic aspects data of T2DM patients and control groups

\begin{tabular}{|l|l|c|c|c|}
\hline \multicolumn{2}{|l|}{ Demographic } & T2DM group (n= 75) & Control group (n= 70) & P-value \\
\hline \multicolumn{2}{|l|}{ Age $($ Mean \pm SE Years) } & $52.30 \pm 1.60$ & $48.88 \pm 0.64$ & 0.08 \\
\hline \multirow{2}{*}{ Gender } & Male & 38 & $35(50.0)$ & 1.0 \\
\cline { 2 - 5 } & Female & 37 & $35(50.0)$ & \\
\hline
\end{tabular}

These findings were in agreement with another previous study that reported that T2DM can be detected at all age groups, So T2DM, diagnosed previously in adults, now impacts children and teenagers ${ }^{(14,15)}$. In contrast, some studies found an inverse relationship between age of onset of T2DM with complication risk and mortality ${ }^{(16,17)}$.

The reasons for age participation in Diabetes have been reported in many studies as by Maedler et al. stated that aging of human pancreatic islets is correlated with decreased proliferation and increased susceptibility to hyperglycemia-induced apoptosis, this represent one explanation for age involvement in diabetes elicition ${ }^{(18)}$. In respect to the gender the current study suggest that there is non-significant $(\mathrm{P}>0.05)$ differences found between the two groups concerning samples distribution according the gender as shown in the table (1). The number and percentage of the female were $37,49.1 \%$ in the patients compared with that in control $35,50 \%$; while of the male were $38,50.6 \%$ in the patients compared with that was in control $35,50 \%$. The result of this study that infer non-significant differences in gender distribution was in agreement with some studies in this domain that refer to" male and female have similar diabetes prevalence biologically"(19). One of explanation for this similarity in overall prevalence in both sex that in T2DM, the deficiency of insulin sensitivity and insulin secretion is significant and identical in both gender ${ }^{(20)}$.

With respect for investigating kidney function in diabetic patients through determining of $B$. urea and $S$. creatinin shown in table (2) and according results of this present study there is a non-significant increase was found in levels of B.urea between two studied groups (T2 DM patients $32.10 \pm 1.60$ control $28.52 \pm 0.84$ ). On the other hand, there is a significant differences in level of $S$. Creatinine between both groups (patients $0.85 \pm$ 0.04 and control $0.71 \pm 0.02$ ), although both groups within the normal range of creatinine. 
Table (2): Urea and Creatinine levels distribution among the studied groups

\begin{tabular}{|l|c|c|c|}
\hline Reneal test & T2DM group $(\mathbf{n}=\mathbf{7 5})$ & Control group $(\mathbf{n}=\mathbf{7 0})$ & $\boldsymbol{P}$-value \\
\hline B,urea & $32.10 \pm 1.60$ & $28.52 \pm 0.84$ & 0.057 \\
\hline S,creatinin & $0.85 \pm 0.04$ & $0.71 \pm 0.02$ & 0.008 \\
\hline
\end{tabular}

The present study results which concern with the levels of urea was agreed and disagreed with some previous studies. Disagreed studies state that $\mathrm{HbA} 1 \mathrm{c}$ and urea were elevated notably in T2DM patients compared to the control (21-23). Another study reported that there was a strong correlation between F.B.S, postprandial blood sugar level and $\mathrm{HbA} 1 \mathrm{c}$ in diabetic patients and urea level(21). The covariance results of our study may be explained by Pathan et al finding who indicates that the period of initiation of T2DM and its severity is strongly associated with an imbalance in serum urea levels, but this state is not similar in case of serum creatinine ${ }^{(24)}$.

With respect to serum creatinine although the control group had a higher values than patients but both group result might be with normal range (below $1.2 \mathrm{mg} \backslash$ dl). A weaker correlation between hyperglycemia and serum creatinine levels was shown according to ${ }^{(22)}$.
The results of our study that concern cholesterol and triglycerides as shown in the table (3) indicate a non-significant raise in cholesterol level between both studied groups (T2 DM patients $177.46 \pm 7.57$ and control $193.34 \pm 2.80$ ).

In contrast, the triglycerides level mean shows a high significant increase $(p<0.01)$ between two groups (T2DM patients $228.12 \pm 20.77$ and control $96.67 \pm$ $1.40)$.

The results of this current study was in agreement with other previous studies that revealed that diabetes was frequently associated with other numerous potential risk factors for cardiovascular problems, like high triglycerides ${ }^{(25,26)}$.

Table (4): Cholesterol and Triglyceride levels distribution among the studied groups

\begin{tabular}{|l|c|c|c|}
\hline Serum lipid & T2DM group $(\mathbf{n}=\mathbf{7 5})$ & Control group $(\mathbf{n}=\mathbf{7 0})$ & $\boldsymbol{P}$-value \\
\hline Cholesterol & $190.84 \pm 7.20$ & $193.34 \pm 2.80$ & 0.755 \\
\hline Triglyceride & $238.62 \pm 19.62$ & $96.67 \pm 1.40$ & $4.72 \times 10^{-10}$ \\
\hline
\end{tabular}

In recent decades, the mean triglyceride was risen in line with the growing epidemic of diabetes mellitus and obesity, but the mean levels of cholesterol in America was reduced $^{(27,28)}$.

Previous studies have indicated that serum triglyceride are positively correlated with diabetes ${ }^{(29,30)}$ since abdominal obesity releases unhealthy free F.A. which are transported to the liver and pancreas contribute to insulin signaling response inhibition or insulin resistance ${ }^{(31)}$.

Less data is available on the relationship between total cholesterol and diabetes. Some studies found that people with abdominal obesity, who respond abnormally to $2 \mathrm{~h} \mathrm{PG}$, associated with high total cholesterol since total cholesterol will increase the dimerization of the endothelial nitric oxide -synthase enzyme, which decreases the activity of glucokinase and thus decreases the glucose intra-cytoplasmic metabolism ${ }^{(32)}$.

In addition to what was mentioned previously, CMV IgG serum level around $82.67 \%$ of T2DM patients are positive for anti-CMV IgG antibody, while only $31.43 \%$ of control are recorded seropositivity for this viral infection, so this difference was high significant ( $p<$ 0.01 ), as shown in figure (1).

The findings of this study suggest that anti-CMV $\mathrm{IgG}$ antibody may be involved in aetiopathogenesis of T2DM and these results are consistent with other studies in this field. One of these studies that reported by Schmidt et al. who indicates that among those who were cytomegalovirus-seropositive, T2DM crude odds were 47 percent higher than cytomegalovirus-seronegative, after adjusting of age and other factors, the correlation 
was greatly reduced and no longer significant, so the correlation between CMV and T2DM is clarified by age and other diabetes risk factors ${ }^{(33)}$.

More frequently, nucleic acids of CMV have been found in diabetic people arterial walls relative to those without diabetes ${ }^{(34)}$. In the pancreata of individuals with T2DM, CMV-RNA has also been identified by ${ }^{(35)}$; However, it is unknown if the pancreatic CMV virus directly affects beta cells and impairs the release of insulin, causing diabetes, or if T2DM patients are a great extent susceptible to CMV infection ${ }^{(35,36)}$.

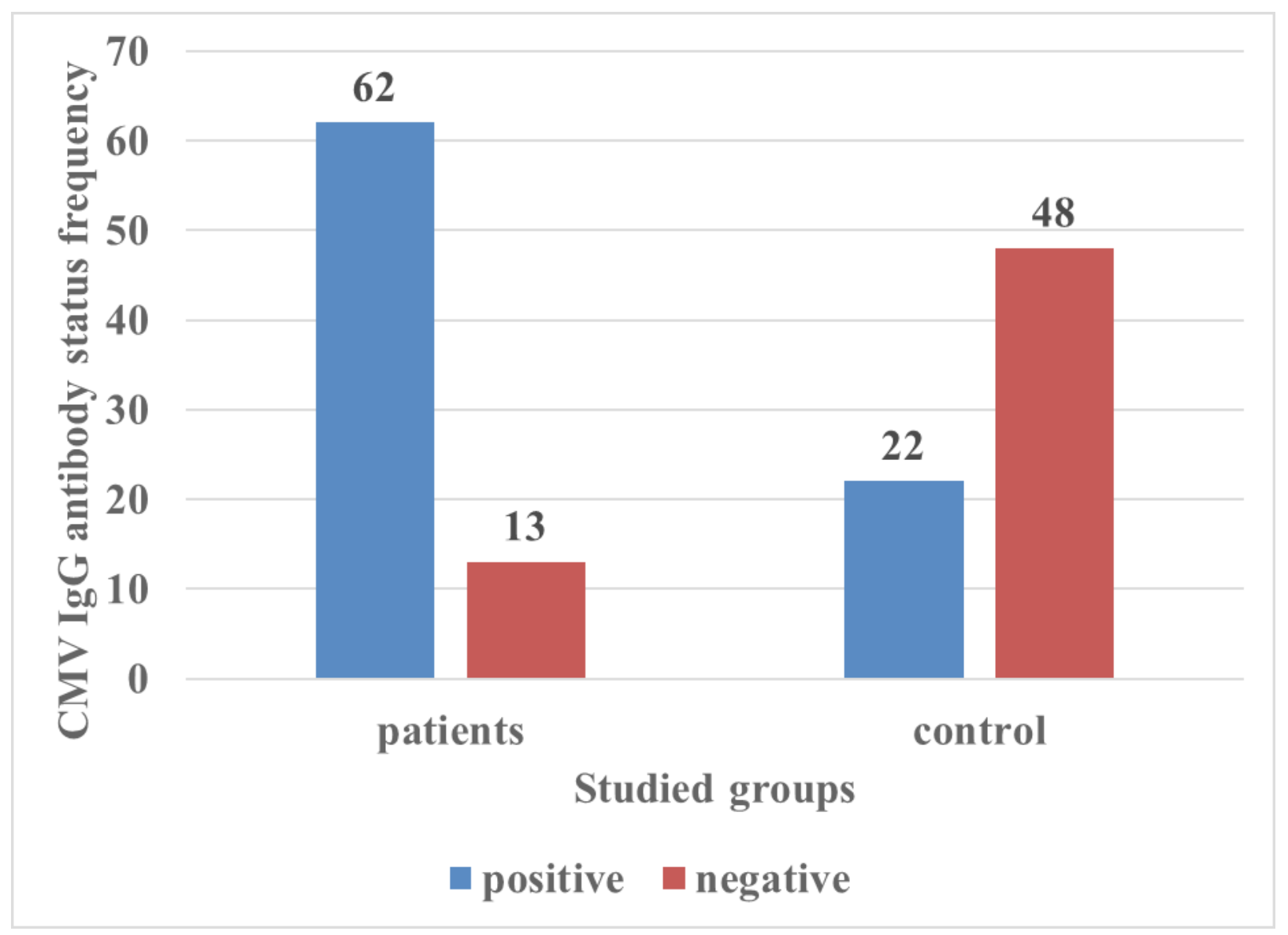

Figure (1): Anti-CMV antibody (IgG) in T2DM group compared to healthy control group

Another potential mechanism that support finding of current study in relation to T2DM elicition by CMV assumes that the molecules used in the structure of the envelope, such as viperin, which is necessary for extracellular budding and complete CMV virion shedding, could modify the pathways of lipid and glucose metabolism ${ }^{(37)}$.

But our study has some limitation like smallest sample size, difficulty for determining if the CMV infection proceed diabetes onest or the diabetic patients are more susceptible for CMV infection because IgG does not show at what time the infection occurred (before or after the emergence of T2DM), but rather demonstrates that an infection occurred at a certain stage in the lifetime of a person ${ }^{(33)}$.

\section{Conclusion}

The viral infection especially CMV infection may have a risk role in etiopathogenesis of T2DM.

Conflict of Interest: None

Funding: Self

Ethical Clearance: Not required 


\section{References}

1. Waddell J. An update on type 2 diabetes management in primary care. Nurse Pract. 2017;42(8):20-9.

2. Tfayli H, Arslanian S. Pathophysiology of type 2 diabetes mellitus in youth: the evolving chameleon. Arq Bras Endocrinol Metabol. 2009;53(2):165-74.

3. Spranger J, Kroke A, Möhlig M, Hoffmann K, Bergmann MM, Ristow M, et al. Inflammatory cytokines and the risk to develop type 2 diabetes: results of the prospective population-based European Prospective Investigation into Cancer and Nutrition (EPIC)-Potsdam Study. Diabetes. 2003;52(3):812-7.

4. Pickup JC. Inflammation and activated innate immunity in the pathogenesis of type 2 diabetes. Diabetes Care. 2004;27(3):813-23.

5. Tilg H, Moschen AR. Inflammatory mechanisms in the regulation of insulin resistance. Mol Med. 2008;14(3-4):222-31.

6. Al-Attaby AKT, Al-Lami MQD. Effects of Duration and Complications of Type 2 Diabetes Mellitus on Diabetic Related Parameters, Adipocytokines and Calcium Regulating Hormones. Iraqi J Sci. 2019;2335-61.

7. Yun J-S, Ko S-H. Risk factors and adverse outcomes of severe hypoglycemia in type 2 diabetes mellitus. Diabetes Metab J. 2016;40(6):423-32.

8. Dunachie S, Chamnan P. The double burden of diabetes and global infection in low and middleincome countries. Trans R Soc Trop Med Hyg. 2019;113(2):56-64.

9. Abu-Ashour W, Twells L, Valcour J, Randell A, Donnan J, Howse P, et al. The association between diabetes mellitus and incident infections: a systematic review and meta-analysis of observational studies. BMJ Open Diabetes Res Care. 2017;5(1).

10. Al-Bayati OHN, Abood WN, Salloom DF. Detection of Epstein Barr Virus Infection in Reactive Arthritis Patients. Iraqi J Sci. 2020;247985.

11. Ouyang Q, Wagner WM, Wikby A, Walter S, Aubert G, Dodi AI, et al. Large numbers of dysfunctional CD8+ T lymphocytes bearing receptors for a single dominant CMV epitope in the very old. J Clin Immunol. 2003;23(4):247-57.

12. Willis EL, Eberle R, WolfRF, White GL, McFarlane
D. The effects of age and cytomegalovirus on markers of inflammation and lymphocyte populations in captive baboons. PLoS One. 2014; 9(9).

13. Nikolich-Žugich J, van Lier RAW. Cytomegalovirus (CMV) research in immune senescence comes of age: overview of the 6th International Workshop on CMV and Immunosenescence. Geroscience. 2017;39(3):245-9.

14. Fagot-Campagna A. Emergence of Type 2 Diabetes Mellitus in Children: Epidemiological Evidence: Division of Diabetes Translation, NationalCenter for Chronic Disease Prevention and Health Promotion, Centers for Disease Control and Prevention, Atlanta, Georgia, USA. J Pediatr Endocrinol Metab. 2000;13(Supplement):1395-402.

15. Ang GY. Age of onset of diabetes and all-cause mortality. World J Diabetes. 2020;11(4):95.

16. Huo L, Magliano DJ, Rancière $F$, Harding JL, Nanayakkara N, Shaw JE, et al. Impact of age at diagnosis and duration of type 2 diabetes on mortality in Australia 1997-2011. Diabetologia. 2018;61(5):1055-63.

17. Al-Saeed AH, Constantino MI, Molyneaux L, D'Souza M, Limacher-Gisler F, Luo C, et al. An inverse relationship between age of type 2 diabetes onset and complication risk and mortality: the impact of youth-onset type 2 diabetes. Diabetes Care. 2016;39(5):823-9.

18. Maedler K, Schumann DM, Schulthess F, Oberholzer J, Bosco D, Berney $\mathrm{T}$, et al. Aging correlates with decreased $\beta$-cell proliferative capacity and enhanced sensitivity to apoptosis: a potential role for Fas and pancreatic duodenal homeobox-1. Diabetes. 2006;55(9):2455-62.

19. Shrestha AD, Kosalram K, Gopichandran V. Gender difference in care of type 2 diabetes. $\mathrm{J}$ Nepal Med Assoc. 2013;52(189).

20. Kautzky-Willer A, Harreiter J, Pacini G. Sex and gender differences in risk, pathophysiology and complications of type 2 diabetes mellitus. Endocr Rev. 2016;37(3):278-316.

21. Abdelsalam KA, AE ME. Correlation between urea level and HbA1c level in type 2 diabetic patients. Sub Med Lab. 2011;1:1-6.

22. Bamanikar SA, Bamanikar AA, Arora A. Study of Serum urea and Creatinine in Diabetic and nondiabetic patients in a tertiary teaching hospital. 
J Med Res. 2016;2(1):12-5.

23. Murugan K, Shrivastava DK, Patil SKB, Sweety L, Debapriya G. Biochemical Investigation of Glycosylated Haemoglobin in Diabetes Associated Nephropathy in Chhattisgarh Population. Pelagia Res Libr Adv Appl Sci Res. 2010;1(2):106-113n.

24. Pathan SB, Jawade P, Lalla P. Correlation of Serum Urea and Serum Creatinine in Diabetics patients and normal individuals. Int $\mathrm{J}$ Clin Biochem Res. 2020;7(1).

25. Kompoti M, Mariolis A, Alevizos A, Kyriazis I, Protopsaltis I, Dimou E, et al. Elevated serum triglycerides is the strongest single indicator for the presence of metabolic syndrome in patients with type 2 diabetes. Cardiovasc Diabetol. 2006;5(1):21.

26. Riediger NDf triglycerides as a predictor of incident, Diabetes insulin resistance and $\beta$-cell function in a, Nation CF, Clark K, Lukianchuk V, Roulette J, et al. Fasting triglycerides as a predictor of incident diabetes, insulin resistance and $\beta$-cell function in a Canadian First Nation. Int J Circumpolar Health. 2017;76(1):1310444.

27. Carroll MD, Lacher DA, Sorlie PD, Cleeman JI, Gordon DJ, Wolz M, et al. Trends in serum lipids and lipoproteins of adults, 1960-2002. Jama. 2005;294(14):1773-81.

28. Flegal KM, Carroll MD, Ogden CL, Curtin LR. Prevalence and trends in obesity among US adults, 1999-2008. Jama. 2010;303(3):235-41.

29. Hjellvik V, Sakshaug S, Strøm H. Body mass index, triglycerides, glucose, and blood pressure as predictors of type 2 diabetes in a middleaged Norwegian cohort of men and women. Clin Epidemiol. 2012;4:213.

30. Tirosh A, Shai I, Bitzur R, Kochba I, Tekes-Manova
D, Israeli E, et al. Changes in triglyceride levels over time and risk of type 2 diabetes in young men. Diabetes Care. 2008;31(10):2032-7.

31. Tchernof A, Després J-P. Pathophysiology of human visceral obesity: an update. Physiol Rev. 2013;

32. Bardini G, Rotella CM, Giannini S. Dyslipidemia and diabetes: reciprocal impact of impaired lipid metabolism and Beta-cell dysfunction on microand macrovascular complications. Rev Diabet Stud RDS. 2012;9(2-3):82.

33. Schmidt L, Nelson HH, Thyagarajan B, Hunter-Schlichting D, Pankow JS, Capistrant $\mathrm{B}$, et al. Association between cytomegalovirus seropositivity and Type 2 diabetes is explained by age and other demographic characteristics: the National Health and Nutrition Examination Survey. Diabet Med. 2018;35(12):1722-6.

34. Lin T-M, Chen WJ, Chen HY, Wang PW, Eng H-L. Increased incidence of cytomegalovirus but not Chlamydia pneumoniae in atherosclerotic lesions of arteries of lower extremities from patients with diabetes mellitus undergoing amputation. J Clin Pathol. 2003;56(6):429-32.

35. Lohr JM, Oldstone MBA. Detection of cytomegalovirus nucleic acid sequences in pancreas in type 2 diabetes. Lancet. 1990;336(8716):644-8.

36. Smelt MJ, Faas MM, de Haan BJ, Draijer C, Hugenholtz GCG, de Haan A, et al. Susceptibility of human pancreatic $\beta$ cells for cytomegalovirus infection and the effects on cellular immunogenicity. Pancreas. 2012;41(1):39-49.

37. Seo J-Y, Cresswell P. Viperin regulates cellular lipid metabolism during human cytomegalovirus infection. PLoS Pathog. 2013;9(8). 